\title{
Interleukin-4 Protects Dopaminergic Neurons In vitro but Is Dispensable for MPTP-Induced Neurodegeneration In vivo
}

\author{
Laura Hühner $^{1 \dagger}$, Jennifer Rilka ${ }^{1+}$, Ralf Gilsbach ${ }^{2}, X_{1 a o l a i}$ Zhou $^{1,3}$, Venissa Machado ${ }^{1}$ and \\ Björn Spittau ${ }^{1 *}$
}

${ }^{1}$ Department of Molecular Embryology, Institute for Anatomy and Cell Biology, Faculty of Medicine, University of Freiburg, Freiburg, Germany, ${ }^{2}$ Institute of Experimental and Clinical Pharmacology and Toxicology, University of Freiburg, Freiburg, Germany, ${ }^{3}$ Department of Molecular Biology and Genetics, Weill Institute for Cell and Molecular Biology, Cornell University, Ithaca, NY, USA

\section{OPEN ACCESS}

Edited by:

Oscar Arias-Carrión Hospital General Dr. Manuel Gea

González, Mexico

Reviewed by:

María Llorens-Martín, Spanish National Research Council,

Spain

Xiaochu Lou,

University of Wisconsin-Madison,

USA

*Correspondence:

Björn Spittau

bjoern.spittau@anat.uni-freiburg.de

${ }^{t}$ These authors have contributed equally to this work.

Received: 05 December 2016 Accepted: 24 February 2017

Published: 09 March 2017

Citation:

Hühner L, Rilka J, Gilsbach R, Zhou X, Machado V and Spittau B (2017) Interleukin-4 Protects Dopaminergic Neurons In vitro but is Dispensable for MPTP-Induced

Neurodegeneration In vivo.

Front. Mol. Neurosci. 10:62. doi: 10.3389/fnmol.2017.00062
Microglia are involved in physiological as well as neuropathological processes in the central nervous system (CNS). Their functional states are often referred to as M1-like and M2-like activation, and are believed to contribute to neuroinflammation-mediated neurodegeneration or neuroprotection, respectively. Parkinson's disease (PD) is one the most common neurodegenerative disease and is characterized by the progressive loss of midbrain dopaminergic (mDA) neurons in the substantia nigra resulting in bradykinesia, tremor, and rigidity. Interleukin 4 (IL4)-mediated M2-like activation of microglia, which is characterized by upregulation of alternative markers Arginase 1 (Arg1) and Chitinase 3 like 3 (Ym1) has been well studied in vitro but the role of endogenous IL4 during CNS pathologies in vivo is not well understood. Interestingly, microglia activation by IL4 has been described to promote neuroprotective and neurorestorative effects, which might be important to slow the progression of neurodegenerative diseases. In the present study, we addressed the role of endogenous and exogenous IL4 during $\mathrm{MPP}^{+}$-induced degeneration of $\mathrm{mDA}$ neurons in vitro and further addressed the impact of IL4-deficiency on neurodegeneration in the 1-methyl-4-phenyl-1,2,3,6tetrahydropyridine (MPTP) mouse model of PD in vivo. Our results clearly demonstrate that exogenous IL4 is important to protect mDA neurons in vitro, but endogenous IL4 seems to be dispensable for development and maintenance of the nigrostriatal system as well as MPTP-induced loss of $\mathrm{TH}^{+}$neurons in vivo. These results underline the importance of IL4 in promoting a neuroprotective microglia activation state and strengthen the therapeutic potential of exogenous IL 4 for protection of mDA neurons in PD models.

Keywords: IL4, MPTP, mDA neuron, microglia, neurodegeneration

\section{INTRODUCTION}

Microglia are the resident immune cells of the central nervous system (CNS), and thus are involved in a plethora of physiological as well as neuropathological conditions (Prinz and Priller, 2014). Similar to peripheral macrophages (Mosser, 2003; Gordon and Martinez, 2010), the functions of microglia are regulated by various endogenous and exogenous stimuli and their functional states 
are referred to as M1-like and M2-like activation (Prinz and Priller, 2014). M1-like microglia activation induced by Th1 cytokines, such as IFN $\gamma$ or the bacterial lipopolysaccharide (LPS), induces secretion of pro-inflammatory cytokines and reactive oxygen species, which are believed to contribute to neuroinflammation-mediated neurodegeneration (Block et al., 2007). M2-like microglia activation is induced by the Th2 cytokines Interleukin 4 (IL4) and Interleukin 13 (IL13) and characterized by upregulation of alternative activation markers Arginase-1 (Arg1) and Chitinase 3 like 3 (Ym1) (Colton and Wilcock, 2010; Zhou et al., 2012). IL4-induced M2-like microglia/macrophage activation has been described to exert neuroprotective effects in mouse models for spinal cord injury (Francos-Quijorna et al., 2016), cerebral ischemia (Liu et al., 2016) and multiple sclerosis (Butti et al., 2008). Next to the abovementioned CNS pathologies, M2-like microglia activation has been further described in animal models of Parkinson's disease (PD) and has been associated with reduced progression and disease severity (Moehle and West, 2015). PD is the second most common neurodegenerative disease and is characterized by the progressive loss of midbrain dopaminergic (mDA) neurons in the substantia nigra (SN) (Jellinger, 2001). This loss of mDA neurons results in a subsequent reduction in striatal dopamine (DA) levels, which is responsible for the classical clinical symptoms such as resting tremor, bradykinesia and rigidity (Jankovic, 2008). Although the IL4-mediated activation of microglia, which is characterized by upregulation of alternative markers Arginase 1 (Arg1) and Chitinase 3 like 3 (Ym1) has been well studied in vitro (Zhou et al., 2012), the role of endogenous IL4 during CNS pathologies in vivo is not well understood. IL4-deficient mice have been reported to develop normally, without showing morphological abnormalities, reduced secretion of IL5 and IL10, as well as an impaired expression of IL4dependent IgE and $\operatorname{IgG}_{1}$ (Metwali et al., 1996). Moreover, loss of IL4 resulted in decreased neuron-microglia communication via the CD200-CD200R ligand-receptor pair and increased neuroinflammatory responses after LPS application (Lyons et al., 2009). CNS-derived IL4 has been further shown to play important roles during regulation of microglia/macrophage activation in the EAE model for multiple sclerosis (Ponomarev et al., 2007). In the present study, we have analyzed the role of endogenous and exogenous IL4 during $\mathrm{MPP}^{+}$-induced degeneration of mDA neurons in vitro and further addressed the impact of IL4-deficiency on neurodegeneration in the 1-methyl-4-phenyl-1,2,3,6-tetrahydropyridine (MPTP) mouse model of $\mathrm{PD}$ in vivo. Our results clearly demonstrate that IL4 is important to protect mDA neurons in vitro but seems to be dispensable for MPTP-induced loss of $\mathrm{TH}^{+}$neurons in vivo.

\section{MATERIALS AND METHODS}

\section{Animals}

C57BL/6 mice were housed at $22 \pm 2^{\circ} \mathrm{C}$ under a $12 \mathrm{~h}$ light/dark cycle with ad libitum access to food and water. C57BL/6-IL4 ${ }^{\text {tm1Nnt }} /$ J mice as described by Metwali et al. (1996) were obtained from the Jackson Laboratory (via Charles River, Germany) and are from now on referred to as IL4 KO mice. All animal procedures were conducted in strict accordance with the German federal animal welfare law, local ethical guidelines and have been approved by the animal experimentation committee of the University of Freiburg and the Regierungspräsidium Freiburg (G-11/77 [MPTP], X-12/01D [E14 mDA neurons], X-12/02D [primary microglia]).

\section{MPTP-Induced Neurodegeneration}

Adult (10-12 weeks) male wild type (WT) and IL4 KO mice were intraperitoneally injected with $20 \mathrm{mg} / \mathrm{kg}$ MPTP hydrochloride (Sigma-Aldrich) dissolved in $0.2 \mathrm{ml}$ phosphate-buffered saline (PBS) once a day for three consecutive days as previously described (Machado et al., 2016a). Mice were sacrificed after 1, 2, 7, and 90 days after injections. PBS only injected mice served as controls. A total of 64 mice were used throughout this study (WT $n=37$, IL4 KO $n=27$ ). All procedures involving MPTP were conducted in strict accordance with published safety and handling guidelines (Przedborski et al., 2001).

\section{Primary Microglia Cultures}

Primary microglia cultures were generated as previously described (Spittau et al., 2013). Briefly, blood vessels and meninges were removed from brains of P0/P1 C57BL/6 mice (Janvier) and brains were washed in ice-cold Hank's BSS (Gibco, Germany). After enzymatic dissociation with Trypsin-EDTA (Gibco, Germany) for $15 \mathrm{~min}$ at $37^{\circ} \mathrm{C}$, an equal volume of fetal calf serum (FCS, Gibco, Germany) together with DNase (Roche, Mannheim, Germany) at a final concentration of $0.05 \mathrm{mg} / \mathrm{ml}$ was added. Cells were dissociated using wideand narrow-bored polished Pasteur pipettes. Finally, cells were centrifuged and resuspended in DMEM/F12 medium (Gibco, Germany) containing 10\% FCS and 1\% penicillin/streptomycin (Invitrogen). Cell suspensions from 2-3 brains were plated on poly-D-lysine-coated (Sigma-Aldrich, Schnelldorf, Germany) $75 \mathrm{~cm}^{2}$ culture flasks. Cultures were kept in a $5 \% \mathrm{CO}_{2} / 95 \%$ humidified atmosphere at $37^{\circ} \mathrm{C}$. After $10-14$ days in culture, microglia were shaken off (250-300 rpm for $1 \mathrm{~h}$ ) from adherent astrocytes and were plated according to the experimental purposes.

\section{E14 Ventral Midbrain-Derived Neuron-Enriched and Mixed Neuron-Glia Cultures}

Neuron-enriched and mixed neuron-glia cultures from embryonic day 14 (E14) ventral midbrains of NMRI mice (Charles River Laboratories Inc.) were generated as described previously (Machado et al., 2016b). Dissected ventral midbrains were dissociated using Trypsin-EDTA (Gibco) for $15 \mathrm{~min}$ at $37^{\circ} \mathrm{C}$. DNase (Roche) was added to a final concentration of $0.05 \mathrm{mg} / \mathrm{ml}$ and after gentle trituration using fire-polished Pasteur pipettes, cell suspensions were centrifuged at $1000 \times g$ for $4 \mathrm{~min}$. Cell pellets were resuspended in DMEM/F12 medium (Gibco) containing either 10\% FCS, 10\% horse serum (HS) and $1 \%$ penicillin/streptomycin (Invitrogen) 
to obtain mixed neuron-glia cultures, or N2 supplement (Invitrogen) and $1 \%$ penicillin/streptomycin (Invitrogen) to obtain neuron-enriched cultures. Both cultures were plated on poly-D-lysine-coated (Sigma-Aldrich) glass coverslips (12 $\mathrm{mm}$ diameter) at a density of 1-2 midbrains/coverslip. On day in vitro 1 (DIV1) medium was refreshed and mixed neuron-glia cultures were maintained for additional 7 days to yield mature cultures. Neuron-enriched cultures were treated from DIV1 according to the experimental design. Cultures were kept in a $5 \% \mathrm{CO}_{2} / 95 \%$ humidified atmosphere at $37^{\circ} \mathrm{C} . \mathrm{MPP}^{+}$(Sigma-Aldrich) treatments were performed as described recently (Spittau et al., 2012). IL4 (10 ng/ml) and IGF-1 (50 ng/ml) were obtained from Peprotech (Germany) and prepared and dissolved according to the manufacturer's instructions.

\section{Neutralization of IL4 In vitro}

Blocking of IL4 in E14 ventral midbrain mixed neuron-glia cultures was achieved using a IL4 neutralizing antibody (IL4nAB) obtained from Ebiosciences (Frankfurt, Germany). Functionality and optimal concentrations were confirmed in IL4-treated primary microglia cultures and the IL4 nAB was used in mixed neuron-glia cultures at a dilution of 1:50.

\section{RNA Isolation and Reverse Transcription}

Total RNA was isolated from primary microglia using TRIzol reagent (Invitrogen, Karlsruhe, Germany) according to manufacturer's instructions. RNAs from $\mathrm{SN}$ and caudate putamen $(\mathrm{CPu})$ were isolated after dissection and transfer to RNA later (Ambion). Tissues were homogenized in peqGOLD TriFast (PeqLab) using a Precellys 24 homogenizer (PeqLab). RNA concentration and quality was determined using the

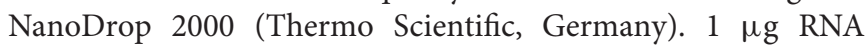
from each sample was reverse transcribed to cDNA using RevertAid (Fermentas, St. Leon-Rot, Germany) according to the manufacturer's instructions.

\section{Semiquantitative and Quantitative RT-PCR}

Semiquantitative RT-PCR (Eppendorf Mastercycler, Eppendorf, Germany) was performed using the PCR Red-Mastermix $2 \mathrm{x}$ (Genaxxon Bioscience, Ulm, Germany). PCR products were separated using agarose gel electrophoresis and visualized after staining with GelRed (Genaxxon Bioscience). Images were captured using a Biometra (Göttingen, Germany) gel documentation station. Quantitative RT-PCR was performed using the $\mathrm{MyiQ}^{\mathrm{TM}}$ system (Bio-Rad, München, Germany) in combination with the Quantitect SYBR Green PCR Kit (Applied Biosystems, Darmstadt, Germany). $1 \mu \mathrm{l}$ of cDNA template was used in $25 \mu \mathrm{l}$ reaction mixture. Results were analyzed using the Bio-Rad iQ5 Optical System Software and the comparative CT method. All data are expressed as $2^{-\Delta \Delta C T}$ for the gene of interest normalized to the housekeeping gene Gapdh and presented as fold change relative to controls. The following primers have been used throughout this study: Arglfor 5'- AACACTCCCCTGACAACCAG-3', Arg1rev 5'-CT
GAAAGGAGCCCTGTCTTG-3' [NM_007482.3], Igflfor 5'-C TGGACCAGAGACCCTTTGC-3', Igf1 rev 5'-GGACGGGGACT TCTGAGTCTT-3' [NM_010512], IL4for 5'-ATTTTGAACGAG GTCACAGGAGAAG-3', IL4rev 5'-ACCTTGGAAGCCCTACA GACGAG-3' [NM_021283.2], Gapdhfor 5'-GGCATTGCTCTC AATGACAA-3', Gapdhrev 5'-ATGTAGGCCATGAGGTCCAC$3^{\prime}$ [NM_001289726].

\section{ELISA}

Interleukin 4 was detected in serum-free culture medium from E14 mixed neuron-glia cultures from control and $\mathrm{MPP}^{+}$-treated groups after $48 \mathrm{~h}$ as well as in medium from primary microglia cultures from control and $\mathrm{MPP}^{+}$-treated groups after $24 \mathrm{~h}$ using the murine IL4 ELISA Development Kit (Peprotech, Hamburg, Germany). IGF-1 was detected in serum-free culture medium from control IL4-treated primary microglia cultures using the murine IGF-1 ELISA Development Kit (Peprotech). ELISAs were performed according to the manufacturer's instructions. Color reactions were performed using 2,2 azinobis(3-ethylbenzothiazoline-6-sulphonic acid) substrate (ABTS, Sigma-Aldrich, Germany) for $30 \mathrm{~min}$ in the dark. Finally, absorbance was detected using Multiskan FC plate reader (Thermo Fischer) at the absorption of $405 \mathrm{~nm}$. Concentrations of IL4 and IGF-1 were calculated from standard curves using the GraphPad Prism5 software (GraphPad Software Inc.).

\section{Immunohistochemistry and Immunocytochemistry}

Numbers of tyrosine hydroxylase (TH) positive neurons in E14 ventral midbrain neuron-enriched as well as mixed neuronglia cultures were determined after immunocytochemistry. After incubation of neuron cultures for different time points, coverslips were washed once with PBS and fixed using 4\% paraformaldehyde in PBS for 15 min at room temperature (RT). Cells were washed three times with PBS for 5 min each, and subsequently blocked and permeabilised for $1 \mathrm{~h}$ at RT in PBS containing $10 \%$ normal goat serum (Invitrogen, Darmstadt, Germany) and $0.1 \%$ Triton-X-100 (Roche Diagnostics). Primary antibodies rabbit anti-TH (1:1.000, polyclonal, Millipore, Schwalbach, Germany), anti-Ibal (1:500, polyclonal, Wako) and anti-Gfap (1:800, monoclonal, Millipore) were incubated overnight at RT. Afterward, coverslips were washed three times with PBS, and cells were incubated with goat-antirabbit peroxidase-conjugated secondary antibodies (1:500, Nordic Immunology) for $1 \mathrm{~h}$ at RT. The immunoreactivity was visualized using diaminobenzidine (Sigma-Aldrich, Deisenhofen, Germany) as described by Adams (1981). Tile scans from whole coverslips were obtained using an Zeiss AxioImager M2 (Zeiss, Göttingen, Germany) and cell counting was performed using ImageJ software (NIH). Immunofluorescent stainings were performed using fixed cultures on glass coverslips as mentioned above, followed by incubation with goat anti-mouse or goat anti-rabbit fluorescence-coupled secondary antibodies (1:200, Cell Signaling Technologies) for $1 \mathrm{~h}$ at RT. The sections were then washed three times with PBS for 3 min each and nuclei were counterstained using 4',6-diamidino-2-phenylindole 
(DAPI, Roche). After a final washing step, sections were placed on objective slides and mounted with Fluoromount G mounting medium (SouthernBiotech). Fluorescence images were captured using the Zeiss AxioImager M2 (Zeiss, Göttingen, Germany).

\section{Unbiased Stereology}

Numbers of $\mathrm{TH}^{+}$mDA neurons, $\mathrm{Ibal}^{+}$microglia and $\mathrm{Gfap}^{+}$ astrocytes were estimated according to the optical fractionator methods using the Stereo Investigator software (MBF Bioscience, Germany). Cell counting was performed using an oil immersion $63 \times$ objective (AxioImager M1, Zeiss, Göttingen, Germany), counting frame of $50 \mu \mathrm{m} \times 50 \mu \mathrm{m}$ and a grid size of $120 \mu \mathrm{m} \times 100 \mu \mathrm{m}$. The optical fractionator was optimized to reach a coefficient of error $\leq 0.1$. For each animal four sections with an evaluation interval of three were selected and labeled cells were counted. The nucleus of each immunoreactive cell was chosen as the counting event. Cell numbers are either presented as total estimated cell numbers or percentages (MPTP model) compared to PBS-treated control animals.

\section{HPLC-Based Quantification of Striatal Dopamine Levels}

Dopamine levels were quantified as recently described (Machado et al., 2016a). Striatal tissues were dissected (Bregma 1.320 ) at 7 and 90 days after MPTP injections, weighted and transferred to ice-cold $0.2 \mathrm{M}$ perchloric acid (40 $\mu \mathrm{l} / \mathrm{mg}$ tissue). Samples were homogenized in $0.2 \mathrm{M}$ perchloric acid using a Precellys 24 tissue homogenizer (Peqlab, Germany). HPLC-ED was performed as described recently (Schneider et al., 2011). Briefly, homogenates were centrifuged at $20.000 \times g$ for $5 \mathrm{~min}$ at $4^{\circ} \mathrm{C}$ and filtered through $0.2 \mu \mathrm{m}$ filters. DA concentrations were detected using a HPLC pump (UltiMate 3000 Quaternary Analytical, Dionex), an autosampler (WISP 717plus, Waters, Germany) and an amperometric detector (Antec Intro, Antec Leyden, Netherlands). The isocratic mobile phase consisted of sodium acetate $(90 \mathrm{mM})$, citric acid monohydrate $(60 \mathrm{mM})$, ethylene- diaminetetraacetic acid (2 mM), 1-octanesulfonic acid ( $3 \mathrm{mM})$, and $8 \%$ methanol and was adjusted to a $\mathrm{pH}$ of 5.3. DA was separated on a Prontosil 120-3-C18 AQ column (3 $\mu \mathrm{m}, 120 \times 2 \mathrm{~mm}$; Bischoff chromatography, Germany) by using a fixed flow rate of $300 \mu \mathrm{l} / \mathrm{min}$. Data were registered and analysed by using Chromeleon 6 software (Dionex).

\section{Statistics}

Data are given as means \pm standard error of the mean (SEM). Statistical differences between two groups were determined using Student's $t$-test. Normality of samples was assessed using the D'Agostino and Pearson omnibus normality test (as recommended by GraphPad Prism) and non-parametric tests have been used where appropriate. Multiple-group analysis was performed using one-way ANOVA followed by Bonferroni's multiple comparison post-test. $P$-values $\leq 0.05$ were considered as statistically significant. All statistical analyses were performed using the GraphPad Prism5 software (GraphPad Software Inc.).

\section{RESULTS}

\section{IL4 Protects mDA Neurons from $\mathrm{MPP}^{+}$-Induced Degeneration in the Presence of Glia Cells}

In order to address the neuroprotective potential of IL4 against $\mathrm{MPP}^{+}$-induced $\mathrm{mDA}$ neurodegeneration, E14 ventral midbrain cultures were treated for $48 \mathrm{~h}$ with $\mathrm{MPP}^{+}(0.2 \mu \mathrm{M})$ in the absence or presence of recombinant IL4 (10 ng/ml). Figures 1A,B demonstrates that neuron-enriched cultures responded to $\mathrm{MPP}^{+}$application with a significant loss of mDA neurons $(39.39 \% \pm 7.821)$. IL4 co-treatment was not sufficient to protect mDA neurons in neuron-enriched cultures from $\mathrm{MPP}^{+}$induced neurodegeneration $(40.59 \% \pm 7.417)$. Next, mDA neurodegeneration was induced by $\mathrm{MPP}^{+}$in mixed neuron-glia cultures. As shown in Figures $\mathbf{1 C}, \mathbf{D}, \mathrm{MPP}^{+}$treatment alone resulted in a significant decrease of $\mathrm{mDA}$ neurons in mixed neuron-glia cultures $(54.93 \% \pm 6.361)$. Interestingly, IL4 cotreatment significantly increased the survival of mDA neurons in mixed neuron-glia cultures $(77.98 \% \pm 4.655)$. These results indicate that IL4 only exerts its neuroprotective effects against $\mathrm{MPP}^{+}$-induced neurodegeneration in the presence of glia cells.

\section{Neutralization of IL4 Increases MPP $^{+}$-Induced Neurodegeneration In vitro}

Since IL 4 has been recently described to be expressed by microglia (Park et al., 2005), we analyzed whether IL4 is expressed in the used E14 mixed neuron-glia culture system. Whereas Gfap ${ }^{+}$ astrocytes were negative for IL4, microglia labeled with FITCcoupled tomato lectin displayed a strong immunoreactivity for IL4 (Figure 2A). Using an IL4 ELISA, we further analyzed the levels of IL4 in supernatants of control- and $\mathrm{MPP}^{+}$-treated cultures. Figure 2B demonstrates that $0.821 \mathrm{ng} / \mathrm{ml}( \pm 0.179)$ IL4 was detectable after $48 \mathrm{~h}$ in supernatants from control cultures and a slight but not significant increase of IL4 levels $(1.123 \mathrm{ng} / \mathrm{ml} \pm 0.5164)$ could be determined after treatment with $\mathrm{MPP}^{+}$. Moreover, we addressed whether primary microglia respond to $\mathrm{MPP}^{+}$treatment with changes in IL4 secretion. $24 \mathrm{~h}$ after treatment with $\mathrm{MPP}^{+}$no change in IL4 secretion from primary microglia cultures was detectable. Interestingly, the data demonstrate that microglia in mixed neuron-glia cultures release higher levels of IL4 than primary microglia. We next aimed to inhibit endogenous IL4 in mixed-neuron glia cultures in order to determine the role of microglia-derived IL4 during $\mathrm{MPP}^{+}$-induced neurodegeneration. Thus, the functionality of a neutralizing IL4 antibody (IL4 nAB) was validated. Therefore, primary microglia cultures were treated with recombinant IL4 $(10 \mathrm{ng} / \mathrm{ml})$ in the presence or absence of IL $4 \mathrm{nAB}$ at dilutions of 1:100 and 1:50, respectively. Using semiquantitative RT-PCR, expression of the IL4-induced M2 marker Arg1 was analyzed. As shown in Figure 2C, Arg1 expression was induced after treatment of primary microglia with IL4 for $24 \mathrm{~h}$. Almost complete abrogation of IL4-triggered Arg1 expression was observed in the presence of the IL4 $\mathrm{nAB}$ at a dilution of 1:50, which was used for further IL4 neutralization experiments. Next, mixed-neuron 

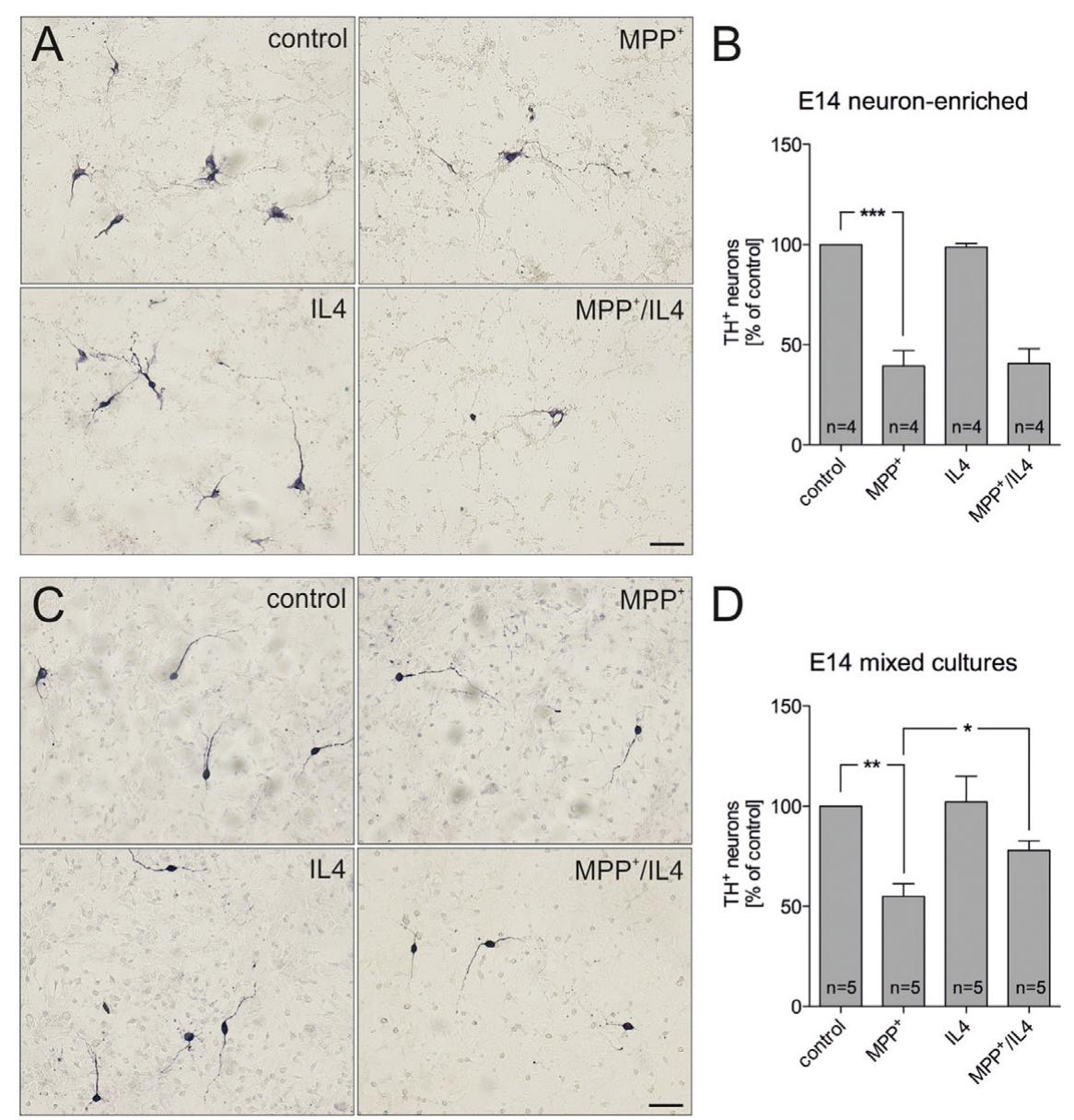

FIGURE 1 | Interleukin 4 (IL4) protects midbrain dopaminergic (mDA) neurons from MPP $^{+}$-induced neurodegeneration in mixed neuron-glia cultures. E14 ventral midbrain neuron-enriched cultures (A) and E14 ventral midbrain mixed neuron-glia cultures (C) were treated with $0.2 \mu \mathrm{M}$ MPP ${ }^{+}$in the presence or absence of IL4 (10 ng/ml) for 2 days and numbers of $\mathrm{TH}^{+}$neurons were counted. Scale bars indicate $50 \mu \mathrm{m}$. Quantifications and statistical evaluations revealed that IL4 failed to protect mDA neurons in E14 neuron-enriched cultures (B) but was sufficient to significantly protect mDA neurons in E14 mixed neuron-glia cultures (D). Data are given as mean \pm SEM from four (neuron-enriched cultures) and five (neuron-glia cultures) independent experiments performed in duplicates. $P$-values derived from one-way ANOVA followed by Bonferroni's multiple comparison post-test are ${ }^{*} p<0.05,{ }^{* *} p<0.01$ and ${ }^{* * *} p<0.001$.

glia cultures were treated with $\mathrm{MPP}^{+}$and endogenous IL4 was blocked (IL4 nAB, 1:50). Figures 2D,E show that inhibition of endogenous IL4 resulted in a significant increase in $\mathrm{MPP}^{+}$induced neurodegeneration $(38.75 \% \pm 6.116)$ as compared to $\mathrm{MPP}^{+}$treatment alone $(54.08 \% \pm 4.72)$. These data clearly demonstrate that microglia-derived IL4 in mixed-neuron glia cultures is important to protect mDA neurons from $\mathrm{MPP}^{+}$ intoxication.

\section{Conditioned Medium from IL4-Treated Primary Microglia Promotes Survival of mDA Neurons}

The abovementioned results indicate that IL4 might shape the functions and properties of microglia in mixed neuron-glia cultures to promote survival of mDA neurons. Hence, primary microglia were treated with IL4 and secretion of cytokines and chemokines were analyzed. As shown in Figure $\mathbf{3 A}$, results of the array using the proteome profiler kit for inflammatory cytokines revealed that IL4 did not influence the secretion of the vast majority of cytokines/chemokines from microglia. Notably, MCP-5 and MIP2 were two candidates with increased secretion after IL4 treatment $(10 \mathrm{ng} / \mathrm{ml})$ for $24 \mathrm{~h}$. We further used microglia-conditioned medium to treat E14 neuron-enriched cultures in order to determine the survival promoting effects of microglia-derived factors on $\mathrm{mDA}$ neurons. Figures $3 \mathbf{B}, \mathbf{C}$ show that microglia-conditioned medium after treatment with IL4 for $24 \mathrm{~h}$ (MCM IL4) significantly increased mDA neuron survival $(157.4 \% \pm 11.13)$ compared to microglia control medium (MCM). The observed neuroprotective effect of MCM IL4 was not promoted by IL4 itself, since direct IL4 treatment of E14 neuron-enriched cultures did not result in an increase of surviving $\mathrm{TH}^{+}$neurons $(108.5 \% \pm 2.849)$. This observation demonstrates that IL4 treatment of microglia induces the secretion of neuroprotective factors, which increase the survival of $\mathrm{TH}^{+}$neurons in E14 neuron-enriched cultures. IGF-1 has 
A
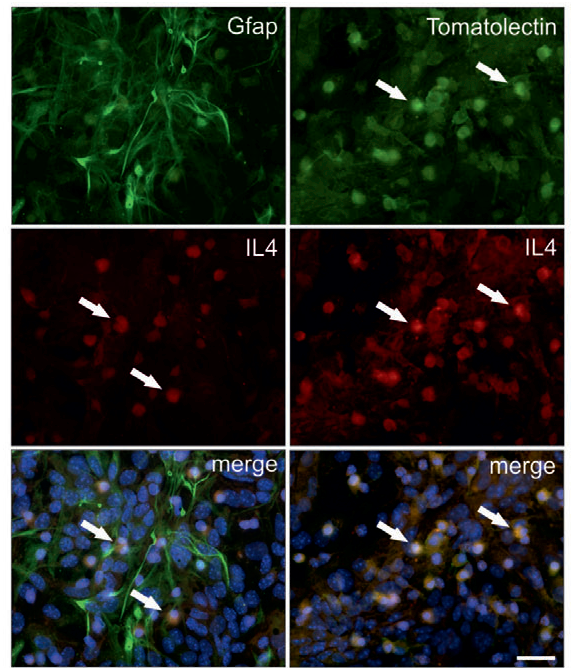

D

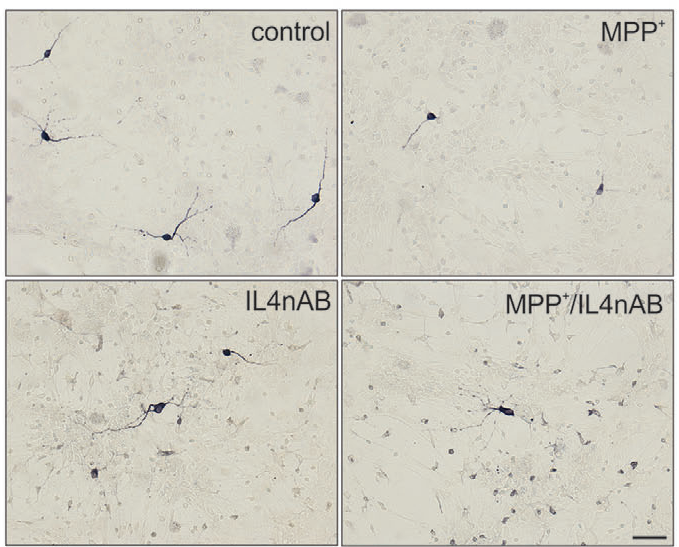

B

$$
\text { E14 mixed cultures microglia cultures }
$$
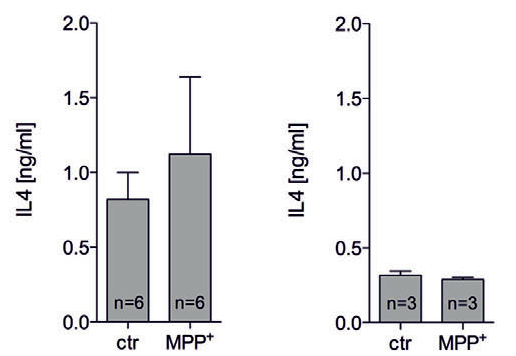

C

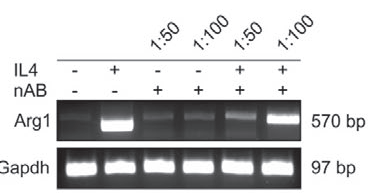

E

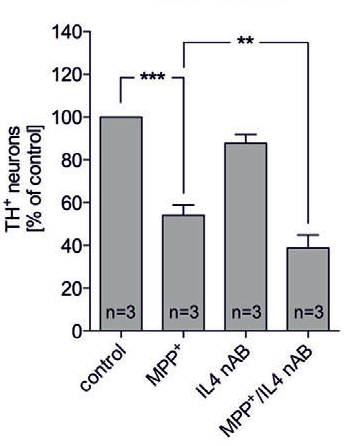

FIGURE 2 | Neutralization of endogenous IL4 increases MPP+'-induced neurodegeneration in vitro. (A) Cellular expression of IL4 in E14 mixed neuron-glia cultures was determined after co-staining with Gfap as an astrocyte marker and FITC-coupled tomato lection as a microglia marker. White arrows mark IL $4^{+}$cells. Scale bar indicates $30 \mu \mathrm{m}$. (B) Secretion of IL4 in E14 mixed neuron-glia cultures under control conditions and 2 days after MPP+ treatment was determined using an IL4 ELISA. The levels of secreted IL4 in supernatants of primary microglia cultures $24 \mathrm{~h}$ after MPP+ treatment $(0.2 \mu \mathrm{M})$ was analyzed using an IL4 ELISA.

(C) Validation of IL4 neutralization in vitro. Primary microglia cultures were treated with recombinant IL4 (10 ng/ml) in the presence (1:50 and 1:100) or absence of an IL4 neutralizing antibody and IL4-mediated increase of Arg1 expression was assessed by PCR. Gapdh was used as housekeeping gene. (D) E14 ventral midbrain mixed neuron-glia cultures were treated with $0.2 \mu \mathrm{M} \mathrm{MPP}^{+}$in the presence or absence of an IL4 neutralizing antibody (IL4nAB) at a dilution of 1:50 for 2 days and numbers of $\mathrm{TH}^{+}$neurons were counted. Scale bars indicate $50 \mu \mathrm{m}$. (E) Quantifications and statistical evaluation demonstrates that neutralization of IL4 increases $\mathrm{MPP}^{+}$-induced degeneration of $\mathrm{mDA}$ neurons in vitro. Data are given as mean \pm SEM from six $\mathbf{( B )}$ and three $\mathbf{( D , E )}$ independent experiments performed in duplicates. $P$-values derived from one-way ANOVA followed by Bonferroni's multiple comparison post-test are ${ }^{* *} p<0.01$ and ${ }^{* * *} p<0.001$.

been identified as a survival-promoting factor secreted by macrophages after IL4 treatment (Wynes et al., 2004). Therefore, we analyzed whether IL4 treatment of primary microglia increases expression and secretion of IGF-1. Using quantitative RT-PCR, we observed that IGF-1 mRNA levels increased after IL4 treatment, reaching a significant peak after $12 \mathrm{~h}$ of treatment (Figure 3D). Moreover, Figure 3E clearly demonstrates that secretion of IGF-1 from primary microglia was significantly increased after $24 \mathrm{~h}(4.919 \mathrm{ng} / \mathrm{ml} \pm 0.2868)$ compared to untreated control cells $(2.061 \mathrm{ng} / \mathrm{ml} \pm 0.3321)$. As IL4 was able to protect $\mathrm{TH}^{+}$neurons from $\mathrm{MPP}^{+}$-induced degeneration in mixed neuron-glia cultures, we wanted to address whether microglia-derived IGF-1 was responsible for this protective effect.
Thus, recombinant IGF-1 was used in order to determine whether $\mathrm{TH}^{+}$neurons in E14 neuron-enriched cultures could be protected from $\mathrm{MPP}^{+}$-induced neurodegeneration. As shown in Figure $3 \mathrm{~F}, \mathrm{MPP}^{+}$treatment significantly reduced the numbers of $\mathrm{TH}^{+}$neurons $(38.2 \% \pm 9.848)$ and a slight non-significant increase of neuron numbers in the presence of IGF-1 could be observed. Although IGF-1 was able to increase the survival of $\mathrm{mDA}$ neurons in the presence of $\mathrm{MPP}^{+}(56.24 \% \pm 10.39)$, the protective effect did not reach significance. Together, these results indicate that IL4 treatment of primary microglia results in the release of protective factors including IGF-1, which might at least partially mediate the neuroprotective properties of microgliaconditioned medium. 


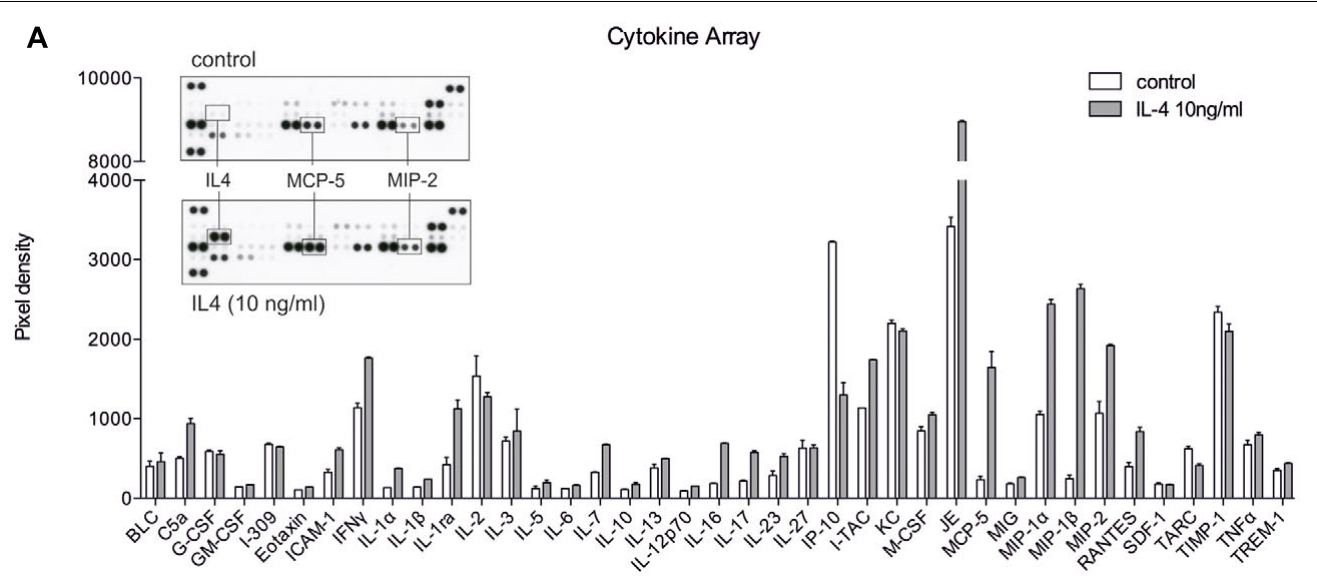

B

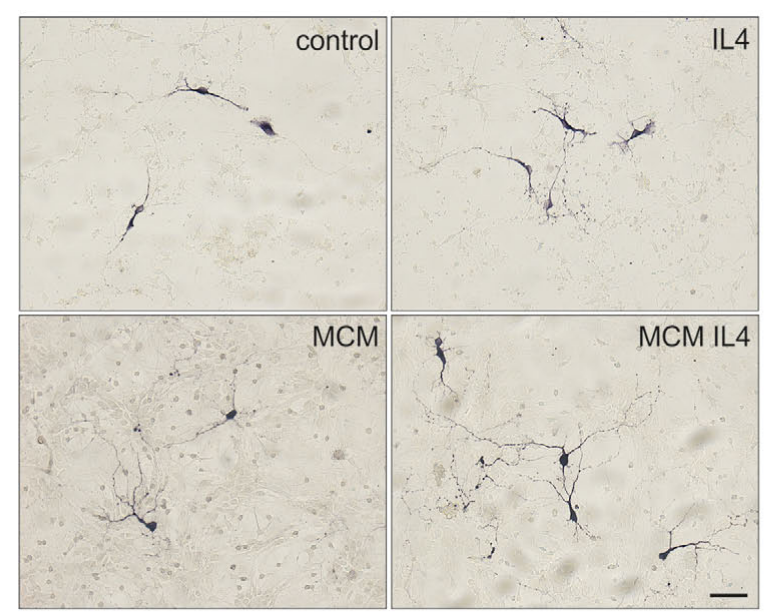

D

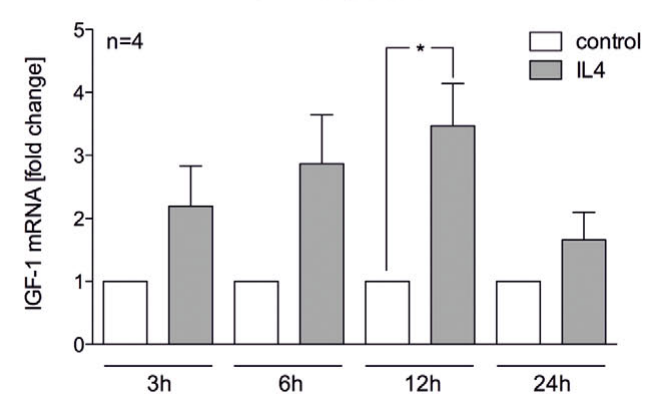

C

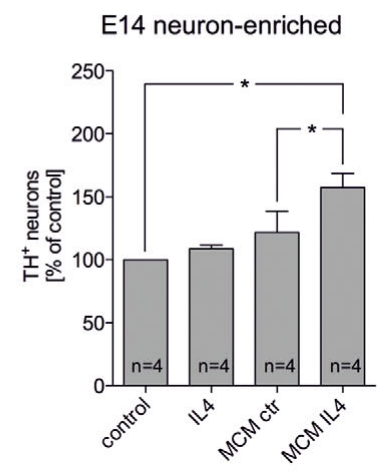
IGF-1 ELISA

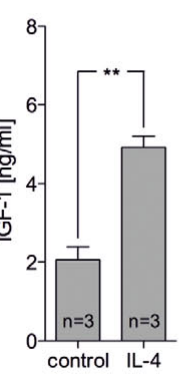

F

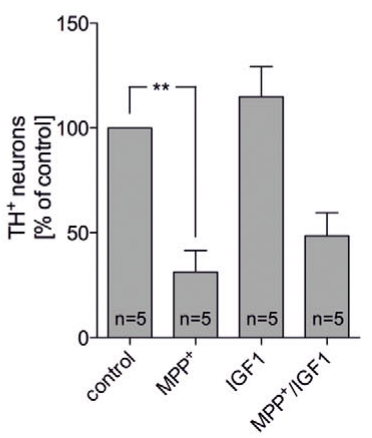

FIGURE 3 | Microglia-conditioned medium after IL4 treatment is neuroprotective in vitro. (A) Treatment of primary microglia with recombinant IL4 (10 ng/ml) for $24 \mathrm{~h}$ results in modest changes of secreted chemokines and cytokines. As expected, IL 4 was detected in IL4-treated samples and thus was not included in the densitometric spot analysis. Levels of MCP-5 and MIP-2 were increased after IL4 treatment. Data are given as mean \pm SEM from two independent experiments. (B) E14 ventral midbrain neuron-enriched cultures were treated for 2 days with serum-free medium (control) and IL4 (10 ng/ml) or with microglia conditioned medium obtained after treatment of primary microglia for $24 \mathrm{~h}$ with serum-free medium (MCM) and IL4 at $10 \mathrm{ng} / \mathrm{ml}$ (MCM IL4). Scale bar indicates $50 \mu \mathrm{m}$. (C) Quantifications of $\mathrm{TH}^{+}$neurons indicate that microglia conditioned medium after treatment with IL4 (MCM IL4) significantly increased neuron survival. IL4 alone was not able to promote neuroprotection. (D) IL4 treatment increases IGF-1 expression in primary microglia. After treatment for 3, 6, 12, and 24 h IGF-1 expression was determined using qPCR and is presented as fold change compared to untreated control cultures. Significant increases in IGF-1 expression was observed after 12 h. (E) IGF-1 secretion under control conditions and $24 \mathrm{~h}$ after treatment with IL4 (10 ng/ml) was detected using an IGF-1 ELISA. (F) Recombinant IGF-1 (50 ng/ml) increased neuron survival in E14 ventral midbrain neuron-enriched cultures without reaching significance. Data are given as mean \pm SEM from four (B-D), three (C, ELISA) and five (E) independent experiments performed in duplicates. $P$-values derived from student's $t$-test (D) are ${ }^{*} p<0.05$ and ** $p<0.01$. $P$-values derived from one-way ANOVA followed by Bonferroni's multiple comparison post-test are ${ }^{*} p<0.05$ and ${ }^{* *} p<0.01$. 

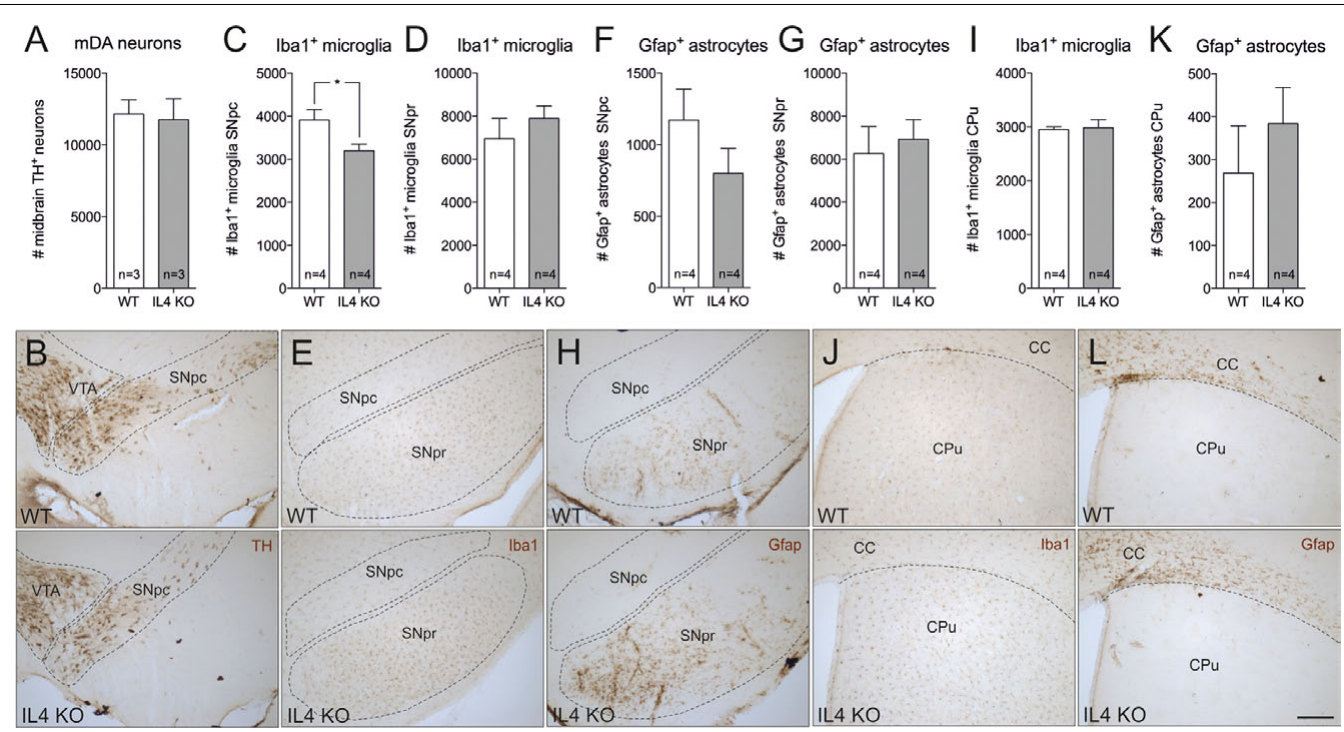

FIGURE 4 | Knockout of IL4 has no effect on the cellular composition of the nigrostriatal system. Changes in numbers of TH ${ }^{+}$neurons (A,B), Iba1 ${ }^{+}$ microglia (C,D,E,I,J) and Gfap ${ }^{+}$astrocytes (F,G,H,K,L) in the nigrostriatal system were analyzed in 4 months old male WT and IL4 KO mice using unbiased stereology. A significant change in cell numbers was only detected for Iba1+ microglia in the SNpc, which were reduced in IL4 KO mice. Scale bar indicates $200 \mu \mathrm{m}$. Data are given as mean \pm SEM from at least three animals per genotype. $P$-values derived from student's $t$-test are * $p<0.05$. VTA, ventral tegmental area; SNpc, substantia nigra, pars compacta; SNpr, substantia nigra, pars reticularis; CC, corpus callosum; CPu, caudate putamen.

\section{Normal Morphology and Cellular Composition of the Nigrostriatal System in IL4-Deficient Mice}

In order to address the role of endogenous IL4 during MPTPinduced degeneration of $\mathrm{mDA}$ neurons in vivo, IL4-deficient mice were used. IL4 KO mice are viable and fertile, develop normally and show no phenotypical abnormalities. However, these mice show impaired secretion of IL5 and IL10 and reduced IL4-dependent expression of IgE and IgG1 (Metwali et al., 1996). As a prerequisite for the use of IL4 KO mice in the MPTP model, we first analyzed the cellular composition of the nigrostriatal system in adult mice. Therefore, 4 months old mice were perfused and brains were cut into $50 \mu \mathrm{m}$ coronal sections, which were subsequently used for TH-, Iba1- and Gfapimmunohistochemistry. Cell numbers were determined using unbiased stereology. As shown in Figures 4A,B, the numbers of $\mathrm{TH}^{+}$mDA neurons in the substantia nigra pars compacta (SNpc) were not altered in IL4-deficient mice. Quantification of Iba $1^{+}$ microglia in SNpc and SNpr revealed a slight and significant decrease in microglia numbers in SNpc (Figures 4C,E) but no impairment of $\mathrm{Iba}^{+}{ }^{+}$microglia numbers in $\mathrm{SNpr}$ (Figures 4D,E). Analysis of $\mathrm{Gfap}^{+}$astrocytes showed that the numbers of reactive astrocytes are neither changed in the SNpc, nor in the SNpr (Figures $4 \mathrm{~F}-\mathrm{H}$ ). Similar results were obtained to the basal ganglia $(\mathrm{CPu})$. The numbers of Iba1 ${ }^{+}$microglia in the CPu of IL4 KO mice were comparable to WT mice (Figures $4 \mathbf{I}, \mathbf{J})$. Numbers of $\mathrm{Gfap}+$ astrocytes in the $\mathrm{CPu}$ were increased in IL4 KO mice without reaching statistical significance (Figures $4 \mathrm{~K}, \mathrm{~L}$ ). These data indicate that loss of IL4 does not impair development and maintenance of the nigrostriatal system in vivo.

\section{Loss of IL4 Does Not Alter the Susceptibility toward MPTP-Induced Neurodegeneration}

Since endogenous IL4 was sufficient to protect mDA neurons from $\mathrm{MPP}^{+}$-induced neurodegeneration in vitro, we used the MPTP mouse model to address the role of IL4 in this model for PD in vivo. Figure 5A displays the injection scheme and the time points used for RNA isolation, immunohistochemistry, and analysis of DA levels using HPLC. We first determined the expression of IL4 in the MPTP model. As shown in Figure 5B, a slight but not significant increase in IL4 expression was observed in the SN 1 day after MPTP injection. All other time points analyzed revealed no increases in IL4 expression in SN or $\mathrm{CPu}$ after intoxication with MPTP. Noteworthy, the relatively high CT values (between 32-36) indicate that in contrast to E14 mixed neuron-glia cultures, IL4 is hardly expressed in the nigrostriatal system in vivo. Two different time points for analysis of $\mathrm{TH}^{+}$neuron numbers and striatal DA levels were chosen according to the acute neurodegeneration phase (7 days) and the regeneration phase (90 days) of this model (Machado et al., 2016c). Figures 5C,E demonstrate that numbers of $\mathrm{TH}^{+}$neurons were significantly reduced in both WT $(57.4 \% \pm 7.895)$ and IL4 KO mice $(56.67 \% \pm 14.39)$ without showing significant differences between both genotypes. Similar results were obtained after analysis of striatal DA levels. IL4 KO mice displayed a significant reduction of DA levels $(46.4 \% \pm 10.51)$ comparable to WT mice $(49.21 \% \pm 3.72$, Figure 5D). Analysis of neuron numbers and DA levels 90 days after MPTP administration revealed that WT and IL4 KO mice showed a similar restoration of the MPTP-intoxicated 
A
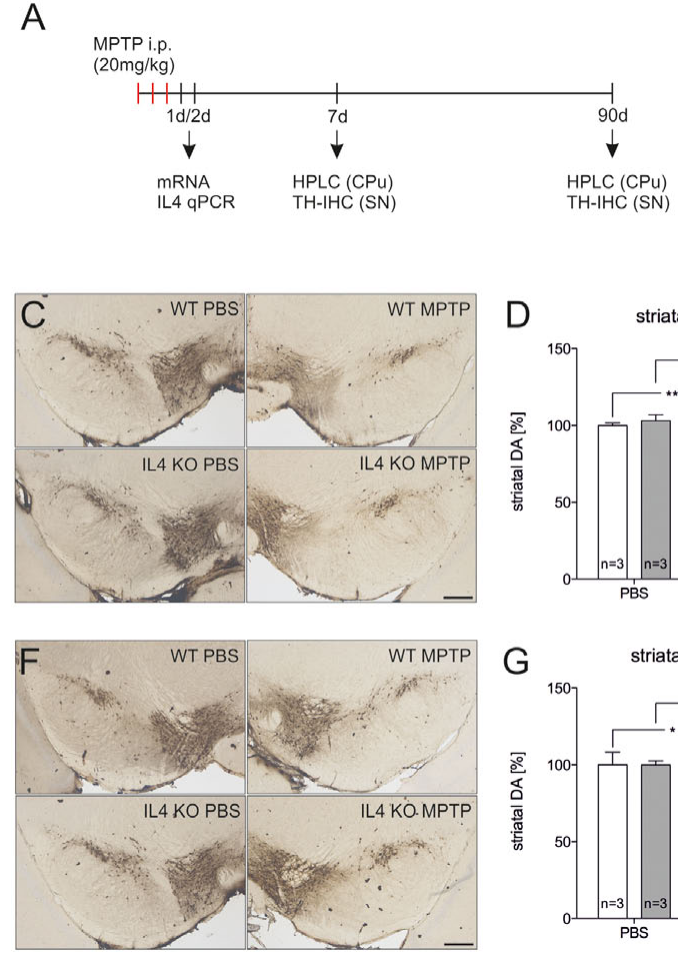
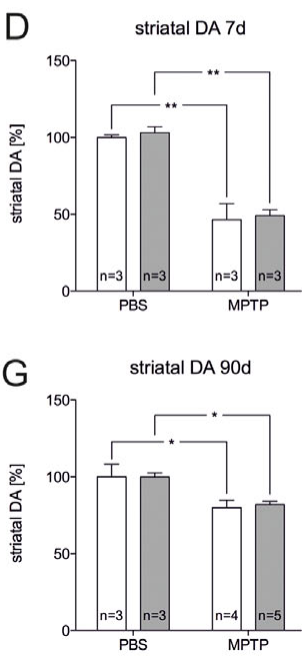

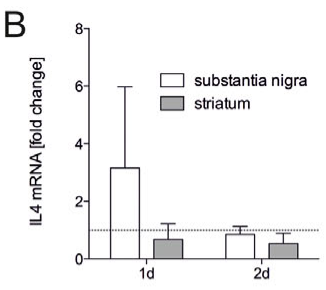

E $\quad \mathrm{TH}^{+}$neurons $7 \mathrm{~d}$

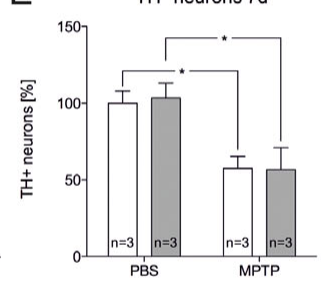

$\square$ WT

$\mathrm{H} \quad \mathrm{TH}^{+}$neurons 90d

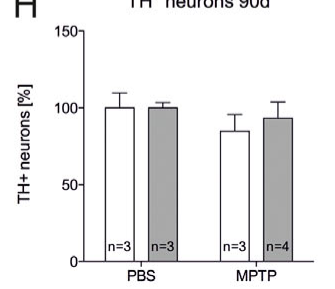

$\square$ WT
$\square$ IL4 KO

FIGURE 5 | Lack of IL4 has no impact on the susceptibility toward 1-methyl-4-phenyl-1,2,3,6-tetrahydropyridine (MPTP)-induced neurodegeneration.

(A) Scheme for MPTP injections and time points used for qPCR, HPLC and immunohistochemistry. (B) Expression of IL4 in total tissue samples from substantia nigra (SN) and caudate putamen (CPu) of wild type (WT) mice 1 and 2 days after MPTP injections. GPCR results were normalized to Gapdh and are given as fold changes ( $n=3$ PBS, $n=3$ MPTP). (C) Immunohistochemical detection of $\mathrm{TH}^{+}$neurons in the SN 7 days after injections with PBS and MPTP. Scale bar indicates $300 \mu \mathrm{m}$. (D) Striatal dopamine levels in PBS- and MPTP-injected mice after 7 days. Equal reductions in dopamine levels were observed in both genotypes.

(E) Quantification of $\mathrm{TH}^{+}$neuron numbers in the SN of PBS- and MPTP-injected mice. No significant changes in neurodegeneration were detected between WT and mutant (IL4 KO) mice. (F) Immunohistochemical detection of $\mathrm{TH}^{+}$neurons in the SN 90 days after injections with PBS and MPTP. (G) Striatal dopamine levels in PBS- and MPTP-injected mice after 90 days. Similar recoveries of dopamine levels were observed in WT and IL4 KO mice. (H) TH+ neuron numbers in the SN of PBS- and MPTP-injected mice after 90 days were not significantly different between WT and IL4 KO mice, indicating normal regeneration of mDA neurons in IL4-deficient mice. Scale bars indicate $300 \mu \mathrm{m}$. Data are given as mean \pm SEM from at least three mice per genotype and time point. $P$-values derived from one-way ANOVA followed by Bonferroni's multiple comparison post-test are ${ }^{*} p<0.05$ and ${ }^{* *} p<0.01$.

nigrostriatal system. As shown in Figures 5F, H, numbers of mDA neurons in the SNpc recovered similarly in WT $(84.82 \% \pm 10.86)$ as well as in IL4 KO mice $(93.29 \% \pm 10.4)$. Moreover, striatal DA levels increased in both genotypes (WT: $79.87 \% \pm 4.956$, IL4 KO: $82.14 \% \pm 1.976)$ compared to 7 days after intoxication, but were still significantly reduced at 90 days compared to PBS-injected control mice (Figure 5G). Taken together, these data demonstrate that loss of endogenous IL4 does not increase the susceptibility toward MPTP-induced degeneration of $\mathrm{mDA}$ neurons in vivo. Moreover, the regeneration of the nigrostriatal system after MPTP administration is not impaired in IL4 KO mice, indicating that endogenous IL4 is dispensable in the MPTP mouse model for PD.

\section{DISCUSSION}

In the present study, we have demonstrated that exogenous IL4 protects $\mathrm{mDA}$ neurons from $\mathrm{MPP}^{+}$-induced degeneration in the presence of glia cells in vitro. Moreover, we provide evidence that endogenous microglia-derived IL4 in mixed neuron-glia cultures is important to reduce $\mathrm{mDA}$ neurodegeneration after $\mathrm{MPP}^{+}$ application and might facilitate neuroprotection by regulating microglia-mediated secretion of neuroprotective factors such as IGF-1. In vivo, the expression of IL4 was hardly detectable under basal conditions or after MPTP administration, and the loss of IL4 did not alter the susceptibility of mDA neurons toward MPTP-induced degeneration. Moreover, the regeneration of the nigrostriatal system after MPTP intoxication, as evident by restoration of $\mathrm{TH}^{+}$neuron numbers as well as striatal DA levels, was not impaired in IL4-deficient mice.

Interleukin 4 has been described to promote a microglia activation state, which is characterized by functional features such as tissue repair and cellular protection (Colton and Wilcock, 2010). Here, we have demonstrated that microglial expression and secretion of cytokines/chemokines is not dramatically altered after IL4 treatment and thus, the neuroprotective effects for $\mathrm{TH}^{+}$neurons seem to be mediated by other microgliaderived factors. However, IL4 has been reported to induce IL6-producing macrophages that inhibit neuroinflammation 
in vitro and in vivo (Casella et al., 2016). Interestingly, IL6 is able to protect $\mathrm{mDA}$ neurons from $\mathrm{MPP}^{+}$-driven degeneration (Spittau et al., 2012) and reduced expression of IL6 has been linked to increased degeneration of mDA neurons after treatment of GDF15-deficient mice with 6OHDA (Machado et al., 2016b). Nevertheless, IL6 was not regulated after treatment of microglia with IL4 in the cell culture model used in this study. Another molecular mechanism to explain IL4-mediated neuroprotection in mixed neuronglia cultures is the regulation of microglia function via the CD200/CD200R ligand/receptor pair. Whereas neurons express CD200, expression of CD200R is restricted to myeloid cells including microglia and binding of CD200 to its cognate receptor reduces microglia activation (Barclay et al., 2002). IL4 has been shown to modulate these interactions by increasing the surface expression of CD200R in microglia (Lyons et al., 2007). Moreover, lack of CD200 results in increased microglia activation after facial nerve transection (Hoek et al., 2000) indicating the importance of this neuron-microglia communication. However, in the present study we observed that microglial conditioned medium after IL4 stimulation is neuroprotective without the physical presence of microglia. This observation suggests that microglia secrete neurotrophic factors after IL4 treatment, which is at least partially responsible for the protection of mDA neurons. We identified IGF-1 as one of these neurotrophic factors and Wynes et al. (2004) have described IL4mediated upregulation of IGF-1 in macrophages. Interestingly, IGF-1 has been reported to protect $\mathrm{mDA}$ neurons in $\mathrm{PD}$ models in vivo. IGF-1 increased survival of $\mathrm{TH}^{+}$neurons and reduced neuroinflammation after intoxication with MPTP (Nadjar et al., 2009) as well as after 6-OHDA injections (Guan et al., 2000). We further tried to rescue mDA neurons from $\mathrm{MPP}^{+}$-induced degeneration, by adding recombinant IGF-1 to neuron-enriched cultures. Although the number of surviving neurons increased, the protection was not as effective as IL4 treatment of mixed neuron-glia cultures. It is high likely that microglia-derived neurotrophic factors and neuronmicroglia interactions contribute to the neuroprotective effects observed after IL4 application in neuron-glia cultures. The fact that IL4 secretion from microglia is not a direct effect of $\mathrm{MPP}^{+}$suggests that challenged neurons in mixed neuronglia cultures trigger IL4 release from microglia. Damaged neurons have been shown not be passive targets of microgliamediated neurotoxicity but are capable of regulating microglia functions. Release of CX3CL1 or IL34 from neurons and the subsequent binding to microglia-specific receptors CX3CR1 and CSF1R are well described mechanisms to shape microglia functions toward a neuroprotective phenotype (Suzumura, 2013). Such neuron-microglia interactions might also be responsible for the IL4 release observed in mixed-neuron glia cultures in the present study and should be further analyzed to understand how $\mathrm{MPP}^{+}$-challenged neurons regulate microglia functions.

In vitro, neutralization of endogenous IL4, expressed by microglia, worsens $\mathrm{MPP}^{+}$-induced degeneration of $\mathrm{mDA}$ neurons. This result suggests that IL4 acts in an autocrine manner on microglia in order to silence their activation. In vivo, loss of IL4 did not change the extent of neurodegeneration and neuroregeneration, indicating that IL4 is dispensable in the MPTP mouse model for PD. One of the reasons for these observations, might be the fact that IL4 expression in the nigrostriatal system is hardly detectable under basal conditions as well as after MPTP intoxication. The main cell type expressing IL4 are Th2 T-cells (Bao and Reinhardt, 2015). However, myeloperoxidase-positive neutrophils after spinal cord injury (Lee et al., 2010) and also damaged neurons (Zhao et al., 2015) are able to express IL4 under neuropathological conditions. The MPTP mouse model for PD is characterized by a distinct leakage of the blood-brain-barrier (BBB) and a modest infiltration of $\mathrm{CD}^{+}$T-cells (Chung et al., 2013) but the neuroinflammatory response is predominantly performed by brain-resident microglia (Depboylu et al., 2012). This is in contrast to the BBB impairment observed in the 6OHDA mouse model for PD, where a pronounced invasion of T-cells and B-cells have been described which makes this model more suitable to study the contribution of adaptive immune responses during $\mathrm{mDA}$ degeneration (Theodore and Maragos, 2015). Lack of appropriate numbers of IL4expressing T-cells might explain why the extent of $\mathrm{mDA}$ neurodegeneration induced by MPTP was not altered in IL4-deficient mice used in the present study. The potential of $\mathrm{CD}^{+}$T-cells to protect $\mathrm{mDA}$ neurons from MPTPinduced neurodegeneration has been described after adoptive transfer of $\mathrm{CD}^{+} / \mathrm{CD} 25^{+}$regulatory T-cells (Reynolds et al., 2007) and highlight the therapeutic options of these antiinflammatory cells. It would be of major interest to address the role of endogenous IL4 in the 6-OHDA mouse model for $\mathrm{PD}$, where the involvement of adaptive immune responses has been clearly demonstrated. Moreover, it has to be taken into account that alternative microglia activation in PD models as evidenced by expression of M2-like markers such as Ym1 and Arg1 (Haas et al., 2016) might be regulated by IL4-independent mechanisms. Contact to apoptotic cells and subsequent release of microglial TGF $\beta 1$ and TGF $\beta 2$ resulting in increased expression of the M2-like marker Arg1 has been described (Spittau et al., 2015). Microglial TGF $\beta$-signaling seems to be essential for promoting alternative microglia activation (Zhou et al., 2012) and could be one mechanism to trigger IL4-independent M2-like microglia activation.

The therapeutic potential of exogenous IL4 has been well documented in several CNS pathology models including models for spinal cord injury (Lee et al., 2010; Francos-Quijorna et al., 2016), APP/PS1 transgenic mice (Kiyota et al., 2010), cerebral ischemia (Zhao et al., 2015; Liu et al., 2016) as well as multiple sclerosis (Butti et al., 2008). In these studies, IL4 was either injected or overexpressed in CNS tissues and promoted strong neuroprotective and anti-inflammatory effects. It has been shown that a heterogeneous induction of the microglia M2a phenotype was observed after central administration of IL4 and contributes to neuroprotection (Pepe et al., 2014). However, further studies have to be performed in order to determine the potential of exogenous IL4 in animal models for PD including MPTP and 6-OHDA. 
Taken together, the results presented in this study demonstrate that IL4 is able to shape microglia functions to promote survival of mDA neurons. We provided evidence that endogenous IL4 is dispensable for MPTP-induced neurodegeneration in vivo but the presence of IL4 in vitro is important to limit degeneration of $\mathrm{TH}^{+}$ neurons after $\mathrm{MPP}^{+}$intoxication. Our results strengthen the hypothesis that IL4-treated microglia are capable of facilitating protection of $\mathrm{mDA}$ neurons and underline the therapeutic potential of IL4 administration in models of PD.

\section{AUTHOR CONTRIBUTIONS}

BS conceived the project. LH, JR, XZ, VM, and BS performed experiments and analyzed the data. RG performed HPLC

\section{REFERENCES}

Adams, J. C. (1981). Heavy metal intensification of DAB-based HRP reaction product. J. Histochem. Cytochem. 29, 775. doi: 10.1177/29.6.7252134

Bao, K., and Reinhardt, R. L. (2015). The differential expression of IL-4 and IL13 and its impact on type-2 immunity. Cytokine 75, 25-37. doi: 10.1016/j.cyto. 2015.05.008

Barclay, A. N., Wright, G. J., Brooke, G., and Brown, M. H. (2002). CD200 and membrane protein interactions in the control of myeloid cells. Trends Immunol. 23, 285-290. doi: 10.1016/S1471-4906(02)02223-8

Block, M. L., Zecca, L., and Hong, J.-S. (2007). Microglia-mediated neurotoxicity: uncovering the molecular mechanisms. Nat. Rev. Neurosci. 8, 57-69. doi: 10. $1038 /$ nrn2038

Butti, E., Bergami, A., Recchia, A., Brambilla, E., Del Carro, U., Amadio, S., et al. (2008). IL4 gene delivery to the CNS recruits regulatory T cells and induces clinical recovery in mouse models of multiple sclerosis. Gene Ther. 15, 504-515. doi: $10.1038 /$ gt.2008.10

Casella, G., Garzetti, L., Gatta, A. T., Finardi, A., Maiorino, C., Ruffini, F., et al. (2016). IL4 induces IL6-producing M2 macrophages associated to inhibition of neuroinflammation in vitro and in vivo. J. Neuroinflamm. 13, 139. doi: 10.1186/s12974-016-0596-5

Chung, Y. C., Kim, Y.-S., Bok, E., Yune, T. Y., Maeng, S., and Jin, B. K. (2013). MMP-3 contributes to nigrostriatal dopaminergic neuronal loss, BBB damage, and neuroinflammation in an MPTP mouse model of Parkinson's disease. Mediators Inflamm. 2013:370526. doi: 10.1155/2013/370526

Colton, C. A., and Wilcock, D. M. (2010). Assessing activation states in microglia. CNS Neurol. Disord. Drug Targets 9, 174-191. doi: 10.2174/ 187152710791012053

Depboylu, C., Stricker, S., Ghobril, J.-P., Oertel, W. H., Priller, J., and Höglinger, G. U. (2012). Brain-resident microglia predominate over infiltrating myeloid cells in activation, phagocytosis and interaction with T-lymphocytes in the MPTP mouse model of Parkinson disease. Exp. Neurol. 238, 183-191. doi: 10.1016/j.expneurol.2012.08.020

Francos-Quijorna, I., Amo-Aparicio, J., Martinez-Muriana, A., and López-Vales, R. (2016). IL-4 drives microglia and macrophages toward a phenotype conducive for tissue repair and functional recovery after spinal cord injury. Glia 64, 2079-2092. doi: 10.1002/glia.23041

Gordon, S., and Martinez, F. O. (2010). Alternative activation of macrophages: mechanism and functions. Immunity 32, 593-604. doi: 10.1016/j.immuni.2010. 05.007

Guan, J., Krishnamurthi, R., Waldvogel, H. J., Faull, R. L., Clark, R., and Gluckman, P. (2000). N-terminal tripeptide of IGF-1 (GPE) prevents the loss of TH positive neurons after 6-OHDA induced nigral lesion in rats. Brain Res. 859, 286-292. doi: 10.1016/S0006-8993(00)01988-0

Haas, S. J., Zhou, X., Machado, V., Wree, A., Krieglstein, K., and Spittau, B. (2016). Expression of Tgf $\beta 1$ and inflammatory markers in the 6-hydroxydopamine mouse model of Parkinson's Disease. Front. Mol. Neurosci. 9:7. doi: 10.3389/ fnmol.2016.00007 analyses. BS wrote the manuscript. All authors have read and approved the final manuscript and further agreed to be accountable for the content of the work.

\section{FUNDING}

This work was funded by grants from the Deutsche Forschungsgemeinschaft (DFG).

\section{ACKNOWLEDGMENT}

The authors thank Susanna Glaser and Ludmila Butenko for excellent technical assistance.

Hoek, R. M., Ruuls, S. R., Murphy, C. A., Wright, G. J., Goddard, R., Zurawski, S. M., et al. (2000). Down-regulation of the macrophage lineage through interaction with OX2 (CD200). Science 290, 1768-1771. doi: 10.1126/science. 290.5497.1768

Jankovic, J. (2008). Parkinson's disease: clinical features and diagnosis. J. Neurol. Neurosurg. Psychiatry 79, 368-376. doi: 10.1136/jnnp.2007.131045

Jellinger, K. A. (2001). The pathology of Parkinson's disease. Adv. Neurol. 86, 55-72. Kiyota, T., Okuyama, S., Swan, R. J., Jacobsen, M. T., Gendelman, H. E., and Ikezu, T. (2010). CNS expression of anti-inflammatory cytokine interleukin4 attenuates Alzheimer's disease-like pathogenesis in APP+PS1 bigenic mice. FASEB J. 24, 3093-3102. doi: 10.1096/fj.10-155317

Lee, S. I., Jeong, S. R., Kang, Y. M., Han, D. H., Jin, B. K., Namgung, U., et al. (2010). Endogenous expression of interleukin-4 regulates macrophage activation and confines cavity formation after traumatic spinal cord injury. J. Neurosci. Res. 88, 2409-2419. doi: 10.1002/jnr.22411

Liu, X., Liu, J., Zhao, S., Zhang, H., Cai, W., Cai, M., et al. (2016). Interleukin-4 is essential for microglia/macrophage M2 polarization and long-term recovery after cerebral ischemia. Stroke 47, 498-504. doi: 10.1161/STROKEAHA.115. 012079

Lyons, A., Downer, E. J., Crotty, S., Nolan, Y. M., Mills, K. H. G., and Lynch, M. A. (2007). CD200 ligand receptor interaction modulates microglial activation in vivo and in vitro: a role for IL-4. J. Neurosci. 27, 8309-8313. doi: 10.1523/ JNEUROSCI.1781-07.2007

Lyons, A., McQuillan, K., Deighan, B. F., O’Reilly, J.-A., Downer, E. J., Murphy, A. C., et al. (2009). Decreased neuronal CD200 expression in IL-4-deficient mice results in increased neuroinflammation in response to lipopolysaccharide. Brain Behav. Immun. 23, 1020-1027. doi: 10.1016/j.bbi.2009.05.060

Machado, V., Gilsbach, R., Das, R., Schober, A., Bogatyreva, L., Hauschke, D., et al. (2016a). Gdf-15 deficiency does not alter vulnerability of nigrostriatal dopaminergic system in MPTP-intoxicated mice. Cell Tissue Res. 365, 209-223. doi: 10.1007/s00441-016-2406-x

Machado, V., Haas, S. J.-P., von Bohlen Und Halbach, O., Wree, A., Krieglstein, K., Unsicker, K., et al. (2016b). Growth/differentiation factor-15 deficiency compromises dopaminergic neuron survival and microglial response in the 6-hydroxydopamine mouse model of Parkinson's disease. Neurobiol. Dis. 88, 1-15. doi: 10.1016/j.nbd.2015.12.016

Machado, V., Zöller, T., Attaai, A., and Spittau, B. (2016c). Microglia-mediated neuroinflammation and neurotrophic factor-induced protection in the MPTP mouse model of Parkinson's Disease-Lessons from transgenic mice. Int. J. Mol. Sci. 17:E151. doi: 10.3390/ijms17020151

Metwali, A., Elliott, D., Blum, A. M., Li, J., Sandor, M., Lynch, R., et al. (1996). The granulomatous response in murine Schistosomiasis mansoni does not switch to Th1 in IL-4-deficient C57BL/6 mice. J. Immunol. 157, 4546-4553.

Moehle, M. S., and West, A. B. (2015). M1 and M2 immune activation in Parkinson's Disease: foe and ally? Neuroscience 302, 59-73. doi: 10.1016/j. neuroscience.2014.11.018

Mosser, D. M. (2003). The many faces of macrophage activation. J. Leukoc. Biol. 73, 209-212. doi: 10.1189/jlb.0602325 
Nadjar, A., Berton, O., Guo, S., Leneuve, P., Dovero, S., Diguet, E., et al. (2009). IGF1 signaling reduces neuro-inflammatory response and sensitivity of neurons to MPTP. Neurobiol. Aging 30, 2021-2030. doi: 10.1016/j.neurobiolaging.2008. 02.009

Park, K. W., Lee, D. Y., Joe, E. H., Kim, S. U., and Jin, B. K. (2005). Neuroprotective role of microglia expressing interleukin-4. J. Neurosci. Res. 81, 397-402. doi: $10.1002 /$ jnr.20483

Pepe, G., Calderazzi, G., De Maglie, M., Villa, A. M., and Vegeto, E. (2014). Heterogeneous induction of microglia M2a phenotype by central administration of interleukin-4. J. Neuroinflamm. 11:211. doi: 10.1186/s12974014-0211-6

Ponomarev, E. D., Maresz, K., Tan, Y., and Dittel, B. N. (2007). CNS-derived interleukin-4 is essential for the regulation of autoimmune inflammation and induces a state of alternative activation in microglial cells. J. Neurosci. 27, 10714-10721. doi: 10.1523/JNEUROSCI.1922-07.2007

Prinz, M., and Priller, J. (2014). Microglia and brain macrophages in the molecular age: from origin to neuropsychiatric disease. Nat. Rev. Neurosci. 15, 300-312. doi: $10.1038 /$ nrn3722

Przedborski, S., Jackson-Lewis, V., Naini, A. B., Jakowec, M., Petzinger, G., Miller, R., et al. (2001). The parkinsonian toxin 1-methyl-4-phenyl1,2,3,6-tetrahydropyridine (MPTP): a technical review of its utility and safety. J. Neurochem. 76, 1265-1274. doi: 10.1046/j.1471-4159.2001. 00183.x

Reynolds, A. D., Banerjee, R., Liu, J., Gendelman, H. E., and Mosley, R. L. (2007). Neuroprotective activities of CD4+CD25+ regulatory $\mathrm{T}$ cells in an animal model of Parkinson's disease. J. Leukoc. Biol. 82, 1083-1094. doi: 10.1189/jlb. 0507296

Schneider, J., Lother, A., Hein, L., and Gilsbach, R. (2011). Chronic cardiac pressure overload induces adrenal medulla hypertrophy and increased catecholamine synthesis. Basic Res. Cardiol. 106, 591-602. doi: 10.1007/s00395-0110166-z

Spittau, B., Rilka, J., Steinfath, E., Zöller, T., and Krieglstein, K. (2015). TGF $\beta 1$ increases microglia-mediated engulfment of apoptotic cells via upregulation of the milk fat globule-EGF factor 8. Glia 63, 142-153. doi: 10.1002/glia. 22740
Spittau, B., Wullkopf, L., Zhou, X., Rilka, J., Pfeifer, D., and Krieglstein, K. (2013). Endogenous transforming growth factor-beta promotes quiescence of primary microglia in vitro. Glia 61, 287-300. doi: 10.1002/glia.22435

Spittau, B., Zhou, X., Ming, M., and Krieglstein, K. (2012). IL6 protects MN9D cells and midbrain dopaminergic neurons from $\mathrm{MPP}+$-induced neurodegeneration. Neuromol. Med. 14, 317-327. doi: 10.1007/s12017-012-8189-7

Suzumura, A. (2013). Neuron-microglia interaction in neuroinflammation. Curr. Protein Pept. Sci. 14, 16-20. doi: 10.2174/1389203711314010004

Theodore, S., and Maragos, W. (2015). 6-Hydroxydopamine as a tool to understand adaptive immune system-induced dopamine neurodegeneration in Parkinson's disease. Immunopharmacol. Immunotoxicol. 37, 393-399. doi: 10.3109/08923973.2015.1070172

Wynes, M. W., Frankel, S. K., and Riches, D. W. H. (2004). IL-4-induced macrophage-derived IGF-I protects myofibroblasts from apoptosis following growth factor withdrawal. J. Leukoc. Biol. 76, 1019-1027. doi: 10.1189/jlb. 0504288

Zhao, X., Wang, H., Sun, G., Zhang, J., Edwards, N. J., and Aronowski, J. (2015). Neuronal interleukin-4 as a modulator of microglial pathways and ischemic brain damage. J. Neurosci. 35, 11281-11291. doi: 10.1523/JNEUROSCI.168515.2015

Zhou, X., Spittau, B., and Krieglstein, K. (2012). TGF $\beta$ signalling plays an important role in IL4-induced alternative activation of microglia. J. Neuroinflamm. 9:210. doi: 10.1186/1742-2094-9-210

Conflict of Interest Statement: The authors declare that the research was conducted in the absence of any commercial or financial relationships that could be construed as a potential conflict of interest.

Copyright (c) 2017 Hühner, Rilka, Gilsbach, Zhou, Machado and Spittau. This is an open-access article distributed under the terms of the Creative Commons Attribution License (CC BY). The use, distribution or reproduction in other forums is permitted, provided the original author(s) or licensor are credited and that the original publication in this journal is cited, in accordance with accepted academic practice. No use, distribution or reproduction is permitted which does not comply with these terms. 